\title{
The Period-Radius relation: Classical Cepheids and RR Lyrae stars
}

\author{
M. Marconi \\ INAF-Astronomical Observatory of Capodimonte, Via Moiariello 16, \\ 80131 Naples, Italy \\ G. Bono \\ INAF-Astronomical Observatory of Rome, Via Frascati 33, 00040 \\ Monte Porzio Catone, Italy \\ T.E. Nordgren \\ Department of Physics, University of Redlands, 1200 East Colton \\ Avenue, Redlands, CA 92973, USA
}

\begin{abstract}
We present the period-radius relations predicted by nonlinear convective models of Classical Cepheids and RR Lyrae stars. For the former variables we show that the metallicity effect is negligible for both fundamental and first overtone pulsators. This confirms the power of the period-radius relation to estimate Cepheid radii and in turn distances. For the latter class of variables we show that the dispersion of the period-radius relation of both fundamental and first overtone pulsators is significantly reduced once the metallicity dependence is taken into account. This provides a robust method to evaluate the radius of observed RR Lyrae with measured period and metal abundance.
\end{abstract}

\section{Introduction}

Variable stars, such as Classical Cepheids and RR Lyrae, are important not only as distance indicators and tracers of stellar populations, but also to evaluate relevant intrinsic stellar parameters. In particular, the Period-Mass-Radius and the Period-Radius (PR) relations can be easily derived as a result of the basic relation between the pulsation period and the mean stellar density (see Bono et al. 2001b). In this paper we focus our attention on the PR relation obtained for both classes of variable stars on the basis of an updated set of nonlinear, convective pulsation models.

\section{The theoretical PR relation of Classical Cepheids}

The sequences of Cepheid models constructed (Bono, Castellani, \& Marconi 2000; Bono, Marconi, \& Stellingwerf 2000) by adopting the chemical composition typical of the LMC $(\mathrm{Z}=0.008, \mathrm{Y}=0.25)$ have been implemented with new sets of models with stellar masses equal to 4 and $4.25 \mathrm{M}_{\odot}$. The sequences of Cepheid 
models constructed (Bono, Caputo, \& Marconi 2001) by adopting the chemical composition typical of the SMC $(\mathrm{Z}=0.004, \mathrm{Y}=0.25)$ have been implemented with new models covering the mass range $7.15 \leq M / \mathrm{M}_{\odot}<7.45$. Together with these Magellanic Cepheid models we also included the models at solar chemical composition provided by Bono et al. (2001a,b) implemented with a new sequence at $4.75 \mathrm{M}_{\odot}$. Old and new models have been constructed by adopting the same ML relation (Bono et al. 1999). This relation agrees, within the errors, with the more recent one derived by Bono et al. (2000) by weighting evolutionary points within the instability strip, according to the individual evolutionary times along the three subsequent crossings (see Bono et al. 2000 for details). Individual Cepheid radii have been evaluated by averaging the surface radius variation over the pulsation cycle.

The top panel of Fig. 1 shows the predicted quadratic PR relations for the entire set of Galactic and Magellanic Cepheid models pulsating in the fundamental mode. The effect of metal abundance is clearly negligible confirming previous results based on a reduced set of models (see Bono, Caputo, \& Marconi 1998). Data plotted in the bottom panel show linear first overtone PR relations. We note, once again, that the metallicity effect is negligible, thus supporting the use of first overtone pulsators for radius determinations. Moreover, the possibility to derive sound metal-independent radii represents and important result for the application of the Baade-Wesselink techniques that also provide independent distance determinations.
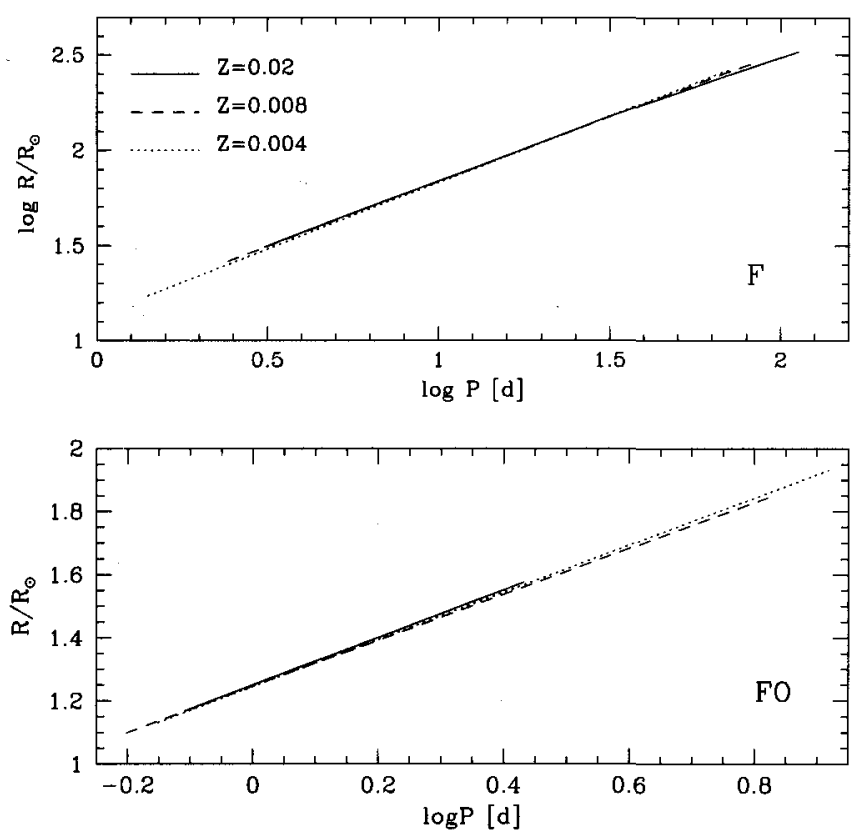

Figure 1. Theoretical PR relation for fundamental (top) and first overtone (bottom) Cepheids at the labeled metallicities. 


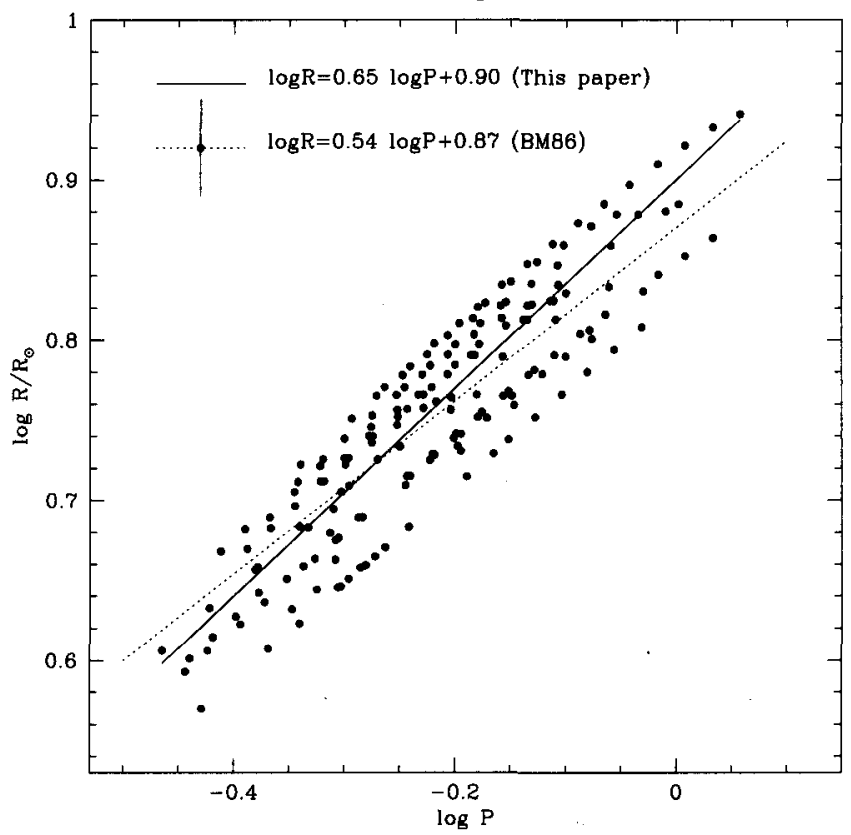

Figure 2. Predicted radii for fundamental pulsators as a function of pulsation periods (see text for details).

\section{The theoretical PR relation for RR Lyrae}

An extensive and homogeneous set of RR Lyrae nonlinear, convective pulsation models, that cover the observed ranges of periods and chemical compositions, has been provided in the last few years (see Bono et al. 2003, and references therein). On the basis of this new theoretical framework we derived accurate radii for fundamental and first overtone RR Lyrae. Fig. 1 shows predicted radii for fundamental pulsators as a function of pulsation periods. The solid line displays the linear regression over the entire set of models, while the dashed line is the empirical PR relation for Type II Cepheids derived by Burki \& Meylan (1986). We notice that the agreement between the empirical relation and current predictions is, within the intrinsic dispersion, quite good. This evidence supports the suggestion by Burki \& Meylan (1986) concerning the similarity of the PR relation between Type II Cepheids and RR Lyrae stars.

This notwithstanding, theoretical radii present, at fixed period, a substantial spread along the best-fit line, thus strongly suggesting the dependence of the $\mathrm{PR}$ relation on a second parameter. We note that the metallicity dependence is expected, since both theory and observations support the evidence that the mean magnitude of RR Lyrae stars depends on this parameter. We performed a linear regression over the entire set of fundamental models and we found the following Period-Radius-Metallicity (PRM) relation:

$$
\log R=0.774( \pm 0.009)+0.580( \pm 0.007) \log P-0.035( \pm 0.001) \log Z
$$

Data plotted in the top panel of Fig. 3 show that the inclusion of the metallicity term causes a decrease in the intrinsic dispersion from 0.03 to $0.008 \mathrm{dex}$. To 
supply a homogeneous theoretical scenario for RR Lyrae radii we also estimated the PRM relation for first overtone pulsators (see Fig. 3, bottom), and we found:

$$
\log R=0.895( \pm 0.012)+0.623( \pm 0.014) \log P-0.026( \pm 0.001) \log Z
$$
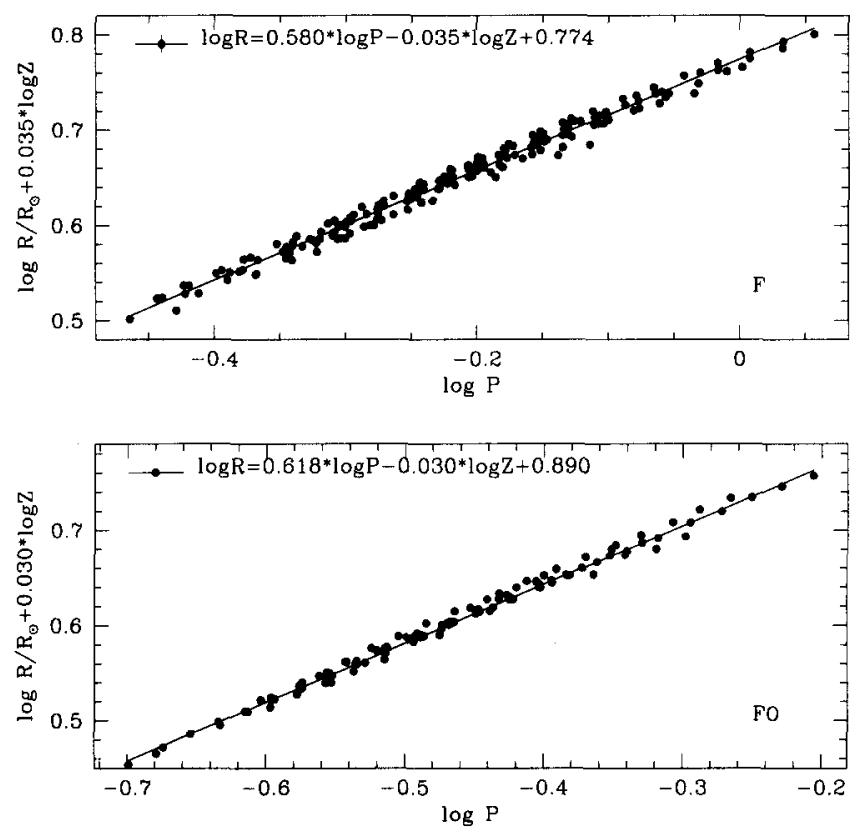

Figure 3. Projected Period-Radius-Metallicity (PRM) relation for fundamental (top) and first overtone (bottom) models.

\section{References}

Bono, G., Caputo, F., Cassisi, S., Marconi, M., Piersanti, L., Tornambé, A. 2000, ApJ, 543, 955

Bono, G., Caputo, F., Castellani, V., Marconi, M., Storm, J., Degl'Innocenti, S. 2003, MNRAS, 344, 1097

Bono, G., Caputo, F., Marconi, M. 1998, ApJ, 497, 43

Bono, G., Caputo, F., Marconi, M. 2001, MNRAS, 325, 1353

Bono, G., Castellani V., Marconi M. 2000, ApJ, 529, 293

Bono, G., Gieren, W. P., Marconi, M., Fouqué, P. 2001a, ApJ, 552, 141

Bono, G., Gieren, W. P., Marconi, M., Fouqué, P., Caputo, F. 2001b, ApJ, 563, 319

Bono, G., Marconi, M., Stellingwerf, R.F. 1999, ApJS, 122, 167

Bono, G., Marconi, M., Stellingwerf, R.F. 2000, A\&A, 360, 245

Burki, G. Meylan, G. 1986, A\&A, 159, 255 